n Phytologia 85(3) we published "A new Zamia (Zamiaceae, Cycadales) from Central Panamá.” In citing the type of the new species, Zamia elegantissima B. Schutzman, A.P. Vovides and R.S. Adams, we neglected to identify and assign the first sheet as the holotype and the second as the isotype, and the International Code of Botanical Nomenclature mandates the explicit identification of the holotype. We validate this new name here by identifying and assigning the holo- and isotypes. The specimen cited as the type of Z. elegantissima is: PANAMA, Colón, Dressler 4897. The holotype is housed at MO, the isotype at PMA. In doing this, the official date of valid publication is the date on which this correction is published.

Zamia elegantissima Schutzman, Vovides et Adams, Phytologia 85(3): 137-145. 1998

HOLOTYPE: PANAMA: Colón, Dressler 4897(MO).

ISOTYPE: (PMA)

Fecha de recepción: 2 de junio de 2004

Versión corregida: 3 de junio de 2004

Aceptado: 3 de junio de 2004

\section{VALIDATION OF THE NAME ZAMIA ELEGANTISSIMA SCHUTZMAN, VOVIDES ET ADAMS}

\author{
Bart Schutzman ${ }^{1}$, Andrew P. Vovides ${ }^{2}$ \\ AND RUSSELL S. ADAMS ${ }^{3}$ \\ ${ }^{1}$ Department of Environmental Horticulture, University of \\ Florida, 200A Mehrhof Hall, \\ Gainesville, Florida 32611-0675, U.S.A. \\ ${ }^{2}$ Instituto de Ecología, A.C., Apartado Postal 63, 91000 Xalapa, \\ Veracruz, México \\ ${ }^{3}$ Gainesville Tree Farm, 15321 N. SR 121, Gainesville, Florida, \\ 32653, U.S.A.
}

E n Phytologia 85(3) publicamos:

E "A new Zamia (Zamiaceae, Cycadales) from Central Panamá". Al citar el tipo de la nueva especie, Zamia elegantissima B. Schutzman, A.P. Vovides and R.S. Adams, omitimos identificar y asignar la primera hoja como el holotipo y la segunda como isotipo. El Código Internacional de Nomenclatura Botánica exige la asignación explícita del holotipo. Por lo tanto validamos este binomio nuevo aquí al identificar y asignar el holotipo y el isotipo a continuación. Al cumplir con esto, la fecha oficial de la publicación válida de este binomio sería la de la publicación de esta corrección.

\section{Zamia elegantissima Schutzman,} Vovides et Adams, Phytologia
85(3): 137-145. 1998

HOLOTIPO: PANAMA: Colón, Dressler 4897(MO).

ISOTIPO: (PMA)

\section{Literature cited}

Schutzman B., Vovides A.P. and Adams R.S. 1998. A new Zamia (Zamiaceae, Cycadales) from Central Panama. Phytologia 85:137-145. 